\title{
Efectos de la Inversión en Infraestructura por el Programa PROMINE/KFW en Centros Educativos
}

\author{
Sandra Meliza Fúnez Cálix ${ }^{1}$ y Mirna Ivette Salgado \\ UNITEC, Tegucigalpa, Honduras.
}

(Enviado: Octubre, 2014 - Publicado: Diciembre, 2014)

\begin{abstract}
Resumen:
La presente investigación se fundamenta en el PROGRAMA PROMINE/KfW, el cual es una donación otorgada por el Gobierno Alemán a través de KfW destinada para contribuir a mejorar la eficiencia y calidad del sistema educativo Pre-básico y Básico en Honduras. La finalidad de este estudio es conocer el efecto de la inversión en la infraestructura por parte del programa PROMINE/KfW en 25 de los 131 centros educativos intervenidos hasta el momento, en seis departamentos (Copan, Intibucá, La Paz, Lempira, Ocotepeque y Santa Bárbara), determinando el monto de la inversión y los cambios realizados en la infraestructura de acuerdo a las necesidades y normas de la Secretaria de Educación. Los departamentos intervenidos son los de mayor índice de pobreza en el país. El programa emplea las metodologías BIOCLIMÁTICO, CPTED y PEC. El tipo de diseño de investigación consiste de un diseño no experimental mixto, utilizando tres tipos de instrumentos: encuestas aplicadas a los directores de los 25 centros, bases de datos obtenidos del FHIS y observación en campo en 5 centros a través de una guía de observación. Utilizando la herramienta estadística SPSS versión 22 para el análisis de variables, se reflejó que 13 de los 25 centros consideran que el mayor beneficiario del programa es la comunidad; los demás centros educativos consideran que los alumnos son los más beneficiados. De todos los encuestados, 19 califican la inversión como excelente. Por lo que se concluye que el Programa ha tenido un impacto positivo en la infraestructura educativa y comunidad en general.
\end{abstract}

\section{Palabras Claves: Bioclimático, CEP, CPTED, PEC, PROMINE/KfW}

\begin{abstract}
:
This research is based on the PROMINE/KfW Program, which is a grant awarded by the German Government through KfW directed to contribute to the improvement of the efficiency and quality of the Pre-Basic and Basic education system in Honduras. The purpose of this study is to determine the effect of the investment in infrastructure of the PROMINE/KfW program in 25 schools out of 131 in six departments (Copan, Intibucá, La Paz, Lempira, Ocotepeque and Santa Barbara), determining the amount of investment and infrastructure changes implemeneted according to the needs and standards of the Ministry of Education. The departments operated are the ones with the highest poverty rate in the country. The program uses the BIOCLIMATIC, CPTED and PEC methodologies. The type of research design consists of a mixed nonexperimental design, using three types of instruments: polls applied to the directors of the 25 centers, data obtained from FHIS and field observation of 5 centers through an observation guide. Using the statistical tool SPSS version 22 for analyzing the variables, 13 of the 25 centers consider that the biggest beneficiary of the program is the community; the other educational centers believe that students benefit the most. Of all the respondents, 19 qualify the investment as excellent. It is concluded that the program has had a positive impact on the educational infrastructure and the community in general.
\end{abstract}

Keywords: Bioclimatic, CEP, CPTED, PEC, PROMINE/KfW

* Las autoras agradecen a Cinthia Isela Caño Acosta y Oscar Cardona Howell, quienes les asesoraron y dieron importantes aportes en la metodología usada para desarrollar este trabajo de investigación y su correspondiente artículo.

\footnotetext{
${ }^{1}$ Autor para correspondencia. Email: sfunezc@unitec.edu
} 


\section{Introducción}

El KfW tiene presencia en Latino América bajo el contexto de cooperación financiera en los países en vías de desarrollo. Inicialmente trabajó con recursos federales para evitar riesgos pero después de cuatro años ha comenzado a mezclar los recursos, siendo el año 2000 el más importante, comenzando con financiamientos mixtos y donaciones. El Programa PROMINE/KfW, es una donación otorgada por el Gobierno Alemán a través del KfW como apoyo al Gobierno de Honduras, destinada al sector educación para contribuir a mejorar la eficiencia y calidad del sistema educativo. La Secretaría de Educación y el Fondo Hondureño de Inversión Social (FHIS) es la institución que administra los recursos para ejecutar los proyectos mediante la modalidad PEC, CPTED y aulas bioclimáticas. (Modernización de la Infraestructura Educativa y de su Gestión Local SE/FHIS VIII, 2011).

La finalidad de la investigación es conocer el efecto de la inversión en infraestructura por parte del Programa PROMINE/KfW, determinar el monto de la inversión, los cambios que se darán en la infraestructura de los centros escolares; conocer el mayor beneficiario de dicha inversión, así como los beneficios obtenidos dentro de la cobertura que tiene $\mathrm{KfW}$. Los efectos de la inversión en la infraestructura, se determinarán de acuerdo a un análisis en los centros educativos. Las comunidades se han integrado a dichos centros para ser administradores y ejecutores de los proyectos, obteniendo efectos positivos en diferentes áreas, tales como el involucramiento en los centros educativos de los padres de familia, alumnos, docentes, así como personas colaboradoras de la comunidad. Esto ha logrado beneficios sociales, educacionales, y de crecimiento personal para los involucrados, llegando éstos a sentirse beneficiados por los proyectos ejecutados.

\section{Marco Teórico}

El Gobierno Alemán a través de KfW apoya al Gobierno Hondureño en el financiamiento del plan "Educación para Todos - Iniciativa de Apoyo Ágil” (EFA/FTI). La Cooperación Alemana es un organismo que proporciona financiamiento reembolsable y no reembolsable a la nación en los sectores de educación, medio ambiente, vivienda y desarrollo económico etc. (SEFIN, 2011).

El 25 de Junio del 2008, KfW suscribió un nuevo convenio de donación con el Gobierno de Honduras con un enfoque a la infraestructura educativa en el área de educación básica denominado "Programa Modernización de la Infraestructura Educativa y su Gestión Local- PROMINE", siendo el beneficiario la Secretaria de Educación (SE) y el Fondo Hondureño de Inversión Social (FHIS), que es la institución que administrará los recursos para ejecutar los proyectos. Esto se hará mediante la modalidad PEC, la cual consiste en realizar proyectos de infraestructura ejecutados por la comunidad. (Convenio de Donación No. 2008 -66- 178).

Las siglas CPTED son una expresión derivada del idioma inglés que significa Crime Prevention Through Environmental Design, en español, Prevención del Delito por Medio del Diseño del Medioambiente (Crime Prevention Through Environmental Design, s.f.).

Las aulas bioclimáticas brindan las condiciones necesarias en las aulas de los centros educativos para un aprendizaje adecuado, tales como elevar la temperatura en zonas frías, bajar la temperatura en zonas con alta temperatura, controlar la humedad relativa del aire 
especialmente en lugares en los que esto sea necesario, contar con buena iluminación y ventilación natural, etc. (Gutiérrez Vera, C., 2012).

El FHIS a través de las alcaldías municipales transfiere los recursos a una cuenta que se apertura por cada proyecto y posteriormente la alcaldía transfiere el dinero al Comité Ejecutor del Proyecto (CEP) a una cuenta abierta en un banco de su comunidad. El CEP es por lo tanto el encargado de administrar, contratar y vigilar la eficiente ejecución de sus proyectos y el FHIS el ente responsable de los procesos operativos y técnicos del Programa. La SE es la encargada de las normas, planificación y priorización de las comunidades que requieren de una intervención en infraestructura educativa y su modernización de acuerdo al plan maestro (Programa PROMINE/KFW, 2009). El PROMINE/KfW, se mantuvo inactivo en el año 2009, debido a la crisis política de Honduras y se reactivó nuevamente en el año 2010. El programa se finaliza en agosto del 2015. El monto de los fondos externos asciende a $€ .18,500.000 .00$, equivalente en L.521, 803,253.11. Actualmente ha comprometido L.393, 567, 691, para la ejecución.

El tema de investigación se basa en 25 proyectos ejecutados y finalizados hasta la fecha, de 131 proyectos que se contemplan realizar una vez que el programa finalice. Se le suma una gestión conjunta entre instituciones para realizar un adecuado uso y mantenimiento de las instalaciones educativas. El Manual PEC se enmarca dentro de la política de descentralización del estado, promoviendo una alta participación ciudadana, con participación de la SE, y el Gobierno Municipal, impulsando el cumplimiento de las iniciativas que deben prevalecer en la concepción, diseño, e implementación del Plan Maestro y redes educativas establecidos por la SE en la Ley de Visión de País y Plan de Nación.

A parte de la metodología CPTED, la Secretaria de Educación y PROMINE han integrado la metodología Bioclimática para los Centros Educativos (CE) a nivel nacional. Se realizaron giras de investigación en las ciudades importantes de la Republica de Honduras, a fin de determinar los diferentes tipos de climas que existen en nuestro país. En base a la información obtenida se identificaron tres clases de climas:

- Clima caliente seco

- Clima caliente húmedo

- Clima frio

En base a los tres climas se concibieron nuevos diseños para las diferentes aulas o módulos que se aplicarán y acondicionaran en los centros educativos de acuerdo a las nuevas normas de la Secretaría de Educación.

\section{Metodología}

El Programa PROMINE/KfW, involucra a la comunidad, sociedad padres de familias, docentes educativos, autoridades municipales, y toda persona que esté involucrada con el centro educativo, para poder llevar a cabo el proyecto mediante la modalidad PEC. La selección de los centros educativos fue definida por la Secretaria de Educación de acuerdo a las redes educativas de los 6 departamentos en los que el Convenio de Cooperación Financiera KfW decidió invertir. Sin embargo el costo de los proyectos que se ejecutarán, se determina mediante la evaluación en campo y posterior formulación por el personal técnico especializado del FHIS. 
El efecto de la inversión del Programa PROMINE/KfW en los centros educativos, se determinó mediante encuesta a directores de 25 centros educativos. Así mismo se realizó una guía de observación en cuatro centros educativos del departamento de la Paz, Municipio de Santa María y un centro educativo en Francisco Morazán, ubicado en Aldea Zambrano, Carretera del Norte de Honduras.

El tipo de diseño es no experimental: cuantitativo, cualitativo, descriptivo. Las técnicas e instrumentos a aplicar están especificadas en la Tabla 1 y las variables de estudio (dependientes e independientes) en la Figura 1.

Tabla 1. Técnicas e instrumentos aplicados

\begin{tabular}{|c|c|}
\hline INSTRUMENTO & CUANDO UTILIZARLO \\
\hline ENCUESTAS & $\begin{array}{l}\text { Preguntas de diferentes tipos: abiertas, cerradas, } \\
\text { escalamiento de Likert }\end{array}$ \\
\hline BASES DE DATOS & $\begin{array}{l}\text { 1. Sistema de costos del FHIS } \\
\text { 2. Sistema financiero de FHIS } \\
\text { 3. Secretaria de Educación } \\
\text { 4. Plan maestro de educación }\end{array}$ \\
\hline GUÍA DE OBSERVACIÓN & $\begin{array}{l}\text { Visitas en campo, se obtuvo información en } \\
\text { cuatro Centros Educativos del Departamento de } \\
\text { La Paz, uno en Francisco Morazán. }\end{array}$ \\
\hline
\end{tabular}

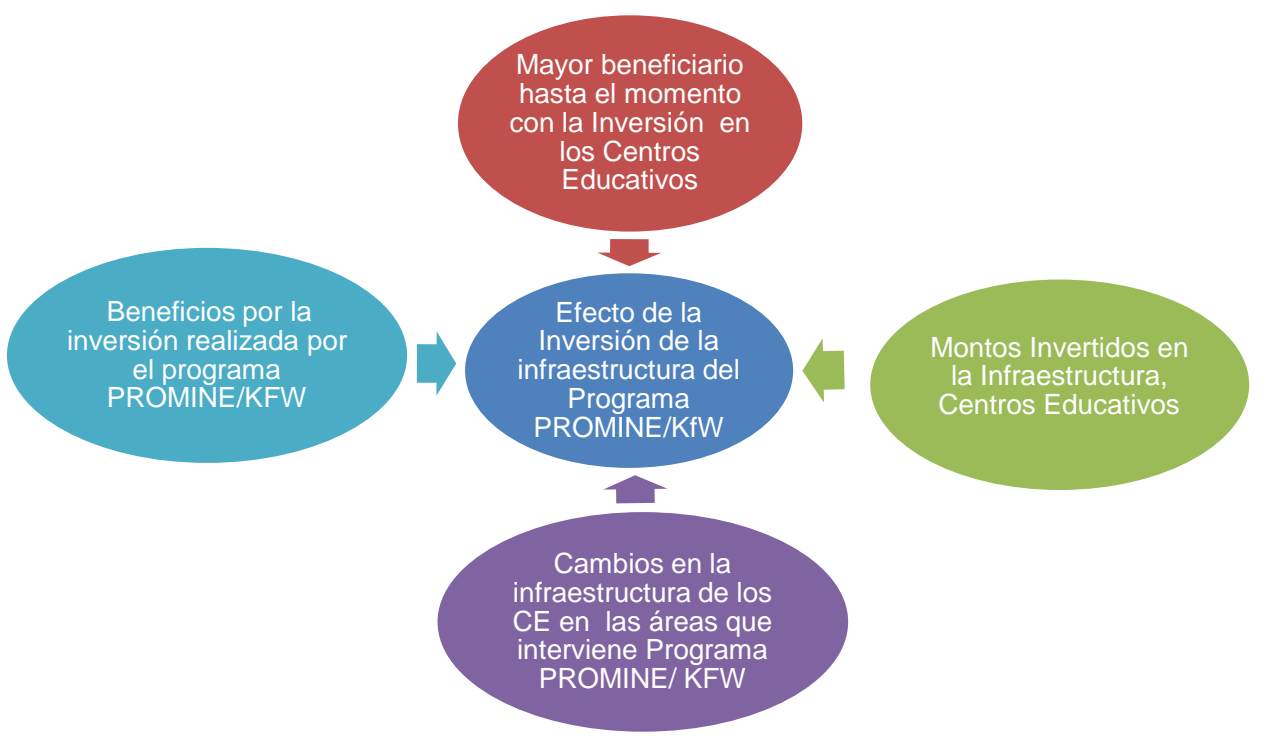

Figura 1. Variables de investigación dependiente e independiente.

Con la información obtenida se podrá realizar un análisis de los datos y conocer el efecto de la inversión realizada mediante la implementación del modelo PEC-FHIS del programa PROMINE/KfW. De acuerdo a las normas y priorización de los centros educativos brindada por la SE, que requieren de una intervención en la infraestructura educativa y su modernización se obtuvo:

- El monto de la inversión de los 25 centros educativos intervenidos.

- Cambios realizados al centro educativo de acuerdo a las normas de la Secretaría de Educación. 
- Grado de satisfacción de la comunidad y docentes de la intervención realizada al centro educativo.

- Determinación del beneficiario del proyecto realizado.

- Grado de importancia para la comunidad del proyecto realizado en el Centro Educativo

\section{Resultados}

Para el análisis de los datos se utilizaron los programas SPSS Statistics para Windows Versión 22.0 y Excel para Windows 2010. Así mismo se usó la base de datos del sistema FHIS obtenidos del Programa PROMINE/KfW en los seis departamentos intervenidos, teniéndose como fuente de información adicional el Plan Maestro de la SE. Se analizó el efecto que el Programa ha tenido en las áreas ya mencionadas.

\subsection{Mayor beneficiario de la inversión del Programa PROMINE /KfW}

Como se observa en la Figura 2, de acuerdo a la investigación realizada, se ha comprobado que en la opinión de los encuestados el mayor beneficiario hasta el momento con la inversión del programa PROMINE/KFW es la comunidad en general.

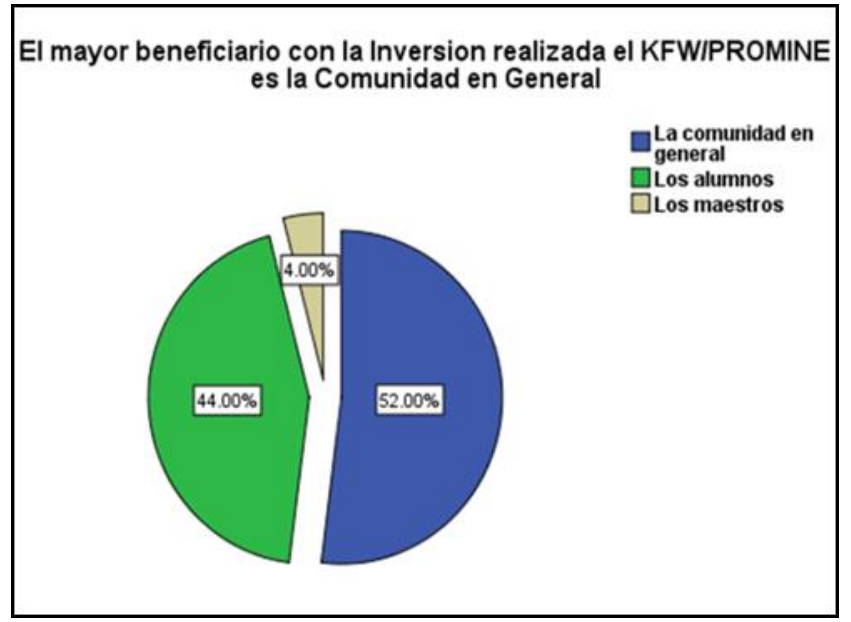

Figura 2. Mayor beneficiario de las instalaciones, por el programa PROMINE/ KfW.

Uno de los factores que influye para tener esta respuesta es que los proyectos son ejecutados mediante la metodología PEC, por lo que la comunidad se involucra en forma directa, junto con los padres de familia y educandos. La junta directiva para desarrollar los proyectos de inversión es conformada por todas las partes antes mencionadas, logrando así un empoderamiento en forma integral para llevar a cabo el plan de acción.

La Modalidad PEC es un instrumento muy valioso, ya que logra que todos los sectores involucrados se sientan parte del proyecto. Debido a que mediante la conformación de los CEP Y CCS se involucra a la comunidad en su proyecto local, se logra un efecto multiplicador bastante positivo. Adicionalmente, al ser las personas capacitadas y entrenadas en cómo realizar y construir el proyecto, se dán efectos colaterales muy importantes para beneficio de la comunidad. Uno de ellos es que las personas aprenden a elaborar trabajos que no habían realizado antes, teniendo así la oportunidad de aprender 
diferentes oficios que a futuro pueden servirles para generar recursos para su núcleo familiar ayudándoles asi a ser auto sostenibles. (KfW-FHIS, 2012.)

\subsection{Montos invertidos en infraestructura del Programa PROMINE/KfW}

Como se observa en la Tabla 2, se obtuvo el monto de inversión realizada por el programa PROMINE/KFW, a partir de la base de datos del FHIS, a través de varias herramientas como ser el Sistema de Costos, Sistema Financiero y Contable, mediante la herramienta informática "Reporteador Financiero".

Tabla.2. Inversión por Departamentos en los 25 centros intervenidos

\begin{tabular}{|l|c|c|}
\hline $\begin{array}{c}\text { Departamentos } \\
\text { Intervenidos }\end{array}$ & $\begin{array}{c}\text { Inversión } \\
\text { (Lempiras) }\end{array}$ & $\%$ \\
\hline Copan & $2,417,159$ & $5 \%$ \\
\hline Intibucá & $14,298,001$ & $27 \%$ \\
\hline La Paz & $16,855,251$ & $32 \%$ \\
\hline Lempira & $9,979,237$ & $19 \%$ \\
\hline Ocotepeque & $5,111,477$ & $10 \%$ \\
\hline Santa Bárbara & $4,465,086$ & $8 \%$ \\
\hline Total & $\mathbf{5 3 , 1 2 6 , 2 1 1}$ & $100 \%$ \\
\hline
\end{tabular}

Se determinó que la mayor inversión se llevó a cabo en el departamento de La Paz, con un monto de 16.8 Millones de Lempiras en las Redes Educativas, seguidamente por el departamento de Intibucá con un valor de 14.3 Millones de Lempiras. Estos montos fueron utilizados en reparaciones, ampliaciones y reposiciones de la infraestructura de los centros educativos.

\subsection{Resumen presupuestos iniciales, órdenes de cambio e inversión final}

La Tabla 3 refleja información por departamento, municipio y aldea, donde el presupuesto aprobado es el resultado de la evaluación realizada por el personal técnico del FHIS. Las órdenes de cambio surgen de algunos imprevistos que surgieron durante la ejecución de la obra, incrementando el presupuesto de la inversión en el centro educativo. La suma de estos dos montos genera la inversión final del programa PROMINE en el centro educativo en cuestión.

Tabla 3. Inversión por Departamentos de los Proyectos realizados por programa.

(Millones de Lempiras)

\begin{tabular}{|l|c|c|c|c|}
\hline \multicolumn{1}{|c|}{ Departamento } & $\begin{array}{c}\text { Centros } \\
\text { Escolares }\end{array}$ & Presupuesto & $\begin{array}{c}\text { Orden de } \\
\text { Cambio }\end{array}$ & $\begin{array}{c}\text { Inversión } \\
\text { Final }\end{array}$ \\
\hline Copan & 2 & L. 2.487 & L. 0.000 & L. 2.417 \\
\hline Intibucá & 6 & L. 12.711 & L. 1.657 & L. 14.298 \\
\hline La Paz & 9 & L. 16.292 & L. 0.633 & L. 16.855 \\
\hline Lempira & 3 & L. 10.118 & L. 0.000 & L. 9.979 \\
\hline Ocotepeque & 3 & L. 5.178 & L. 0.000 & L. 5.111 \\
\hline Santa Bárbara & 2 & L. 4.106 & L. 0.426 & L. 4.465 \\
\hline
\end{tabular}

Las órdenes de cambio que se dieron durante la ejecución del programa, se enuncian a continuación: 
- Carencia de equipo de topografía (estación total), para realizar levantamientos de los centros educativos.

- Los kioskos no se contemplaron dentro del presupuesto aprobado, y posteriormente se consideraron por medio de una orden de cambio

- El equipamiento de los talleres de ciencias naturales, computación, se agregaron mediante ordenes de cambio, para lograr el avance de los proyectos.

- Los huertos escolares, se crearon en una etapa posterior a lo que se contempló inicialmente.

De los 25 proyectos intervenidos, 11 tuvieron un incremento en sus presupuestos, debido a los factores mencionados anteriormente. Se puede apreciar que en los departamentos de Copán, Lempira y Ocotepeque, no se dieron órdenes de cambio en las obras, algo que sí pasó en los departamentos de La Paz, Intibucá, y Santa Bárbara por un monto total de L.2, 716, 530, quedando con una inversión final de L. 53, 126, 211. Esto es un indicativo que la planeación del proyecto sufrió pequeñas modificaciones no significativas, las cuales no generan ningún impacto en cuanto a la disponibilidad de los recursos, ya que se contempla una reserva para cubrir imprevistos.

\subsection{Cambios de infraestructura en los centros educativos de las áreas intervenidas.}

La Tabla 4 muestra la infraestructura de los 25 centros educativos con su infraestructura actual, indicando la cantidad de aulas y módulos a demoler, reparar, y los módulos nuevos realizados posteriormente basados en las necesidades de los centros y de acuerdo a las normas de la SE. La obra final fue realizada aplicando las metodologías de CPTED y aulas bioclimáticas, cubriendo las necesidades de los mismos. (Gutierrez Vera, C., 2012).

Tabla 4. Infraestructura de los 25 centros antes y después de la intervención.

\begin{tabular}{|l|l|l|l|}
\hline \multicolumn{1}{|c|}{ Infraestructura Actual } & \multicolumn{1}{|c|}{$\begin{array}{c}\text { Demoliciones y } \\
\text { Reparaciones }\end{array}$} & \multicolumn{1}{c|}{ Modulos Nuevos } & Obra Final \\
\hline 63 aulas & \multicolumn{1}{|c|}{ Demolición } & 34 Aulas & 88 Aulas \\
\hline 75 Servicio Sanitarios & 9 Aulas & 42 Servicios Sanitarios w/96 & 65 Servicios Sanitarios w/96 \\
\hline 21 Cocinas bodegas & 52 Servicios sanitarios & 6 Modulos de baños de $7 \times 5$ & 6 Modulos de baños de $7 \times 5$ \\
\hline 3 Canchas & 12 Cocinas - bodegas & 2 Modulos de baños $1.85 \times 8$ & 2 Modulos de baños $1.85 \times 8$ \\
\hline 1 Salon de usos multiples & 1 Modulo administrativo & 1 Modulo de $3.5 \times 8$ & 1 Modulo de $3.5 \times 8$ \\
\hline 1 Biblioteca & \multicolumn{1}{|c|}{ Reparaciones } & 1 Biblioteca & 2 Bibliotecas \\
\hline 1 Modulo administrativo & 54 Aulas & 2 Modulo administrativo & 2 Modulo administrativo \\
\hline & 9 Cocinas Bodegas & 7 Modulos de lavamanos & 7 Modulos de lavamanos \\
\hline & 2 Canchas & 13 Cocina - bodega & 22 Cocinas Bodegas \\
\hline & 1 Biblioteca & 3 Canchas Multiusos & 5 Canchas multiusos \\
\hline & 1 Salon de usos multiples & 1 Salon de Usos Multiples & 2 Salon de Usos Multiples \\
\hline & 23 Servcios Sanitarios w/96 & 1 Casa de Maestro & 1 Casa del Maes tro \\
\hline
\end{tabular}

\subsection{Beneficios por la inversión realizada por el Programa PROMINE/KfW}

Hubo un leve incremento del $4.9 \%$ en la matrícula de ciertos centros educativos, de acuerdo al crecimiento poblacional de la región en las áreas rurales.

Se produjo movimiento de 180 Alumnos en matrícula escolar del año 2014, que representa un $10.92 \%$. Estos movimientos se dieron debido a la ejecución de los programas de mejoramiento de los centros educativos, por causa de que los padres buscaban mejores condiciones de seguridad para sus hijos.

El programa PROMINE/KfW incluye un módulo de capacitación a los CEP en las áreas de administración de los recursos financieros, contraloría social, así como la ejecución de 
proyectos "aprendiendo a ser auto sostenibles". Estos módulos no sólo sirven para dar mantenimiento y sostenibilidad a los centros, sino que a su vez le sirve a la comunidad para mejorar su vida personal, obteniendo un valor agregado al aprender a realizar trabajos que les generen ingresos adicionales a sus hogares.

Los centros educativos son utilizados para desarrollar diferentes actividades tanto por los docentes, padres de familia y la comunidad. Ejemplos son capacitaciones de docentes, campañas de vacunación, asambleas de las juntas de aguas y patronatos, encuentros deportivos de jóvenes, etc.

Guías de observación en los sitios de visita.

Se realizó visita a cinco centros educativos, desarrollando una guía de observación en los cuatro Centros del departamento de la Paz, Municipio de Santa María y un Centro Educativo en Francisco Morazán, ubicado en Aldea Zambrano. A través de la visita de campo, se puede apreciar la situación del antes y después de la infraestructura, lo cual muestra el impacto en el mejoramiento de la infraestructura, así como las condiciones óptimas para impartir las clases desde el punto de vista pedagógico.

A continuación una de las obras, Escuela Marco Aurelio Soto, ubicada en Francisco Morazán, aldea Zambrano.

Antes

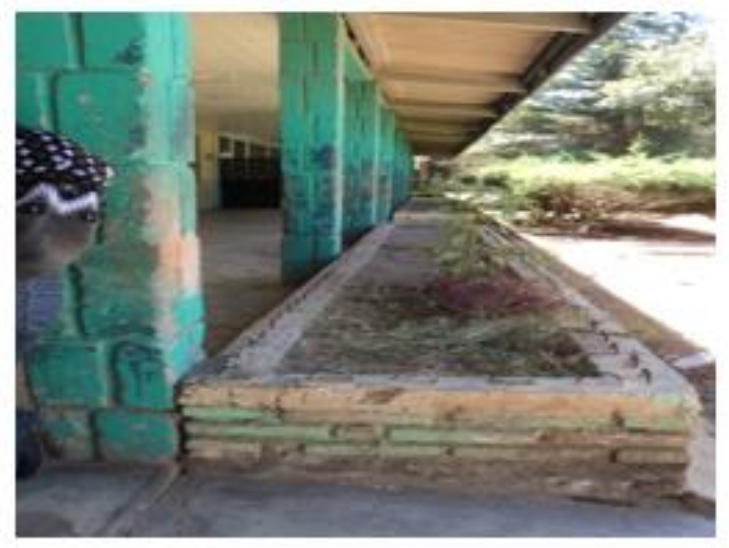

Después

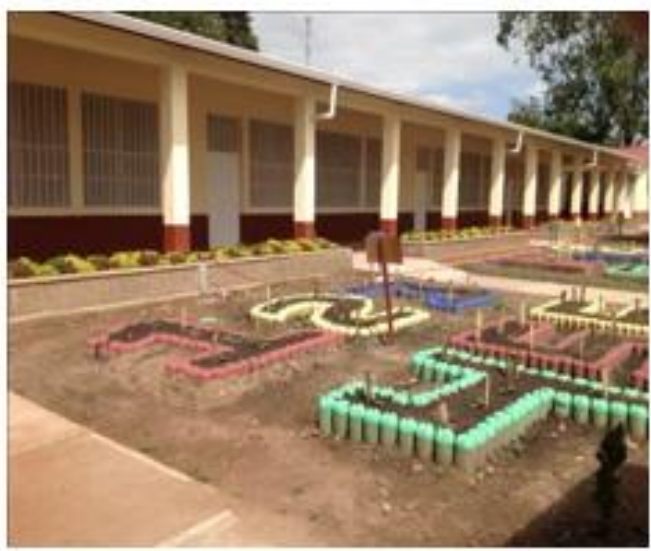

Figura 3. Situación comparativa infraestructural de la Escuela Marco Aurelio Soto.

Comparación entre el sistema constructivo tradicional y el PEC-CPTED Bioclimático.

En otra visita realizada a la Escuela General Francisco Morazán, ubicada en el barrio Las Galeras, Municipio de Santa Bárbara, departamento del mismo nombre, y con código 105092, el Centro Educativo se evaluó, haciéndose el levantamiento de las obras existentes. La evaluación inició cerca de las nueve de la mañana, con un clima agradable a pesar de encontrarse en una zona bastante cálida. A medida que el día avanzó, la temperatura incrementó llegando a cerca de $\operatorname{los} 32^{\circ} \mathrm{C}$. El centro educativo tiene aulas con techo de lámina de asbesto y aluzinc, las cuales se encuentran en mal estado, por lo que se sugirió realizar el cambio de la lámina e incluir a la estructura cielo falso.

Los centros educativos que han sido intervenidos por el Programa PROMINE/KfW, han utilizado diseños Bioclimáticos. Una de las consideraciones que el programa tiene es la 
de contemplar para el techo el uso de lámina aluzinc esmaltada con aislante térmico y cielo falso. Lo anterior hace que las aulas mantengan una temperatura agradable, tanto en la época cálida como fría.

\section{Construcción Tradicional}

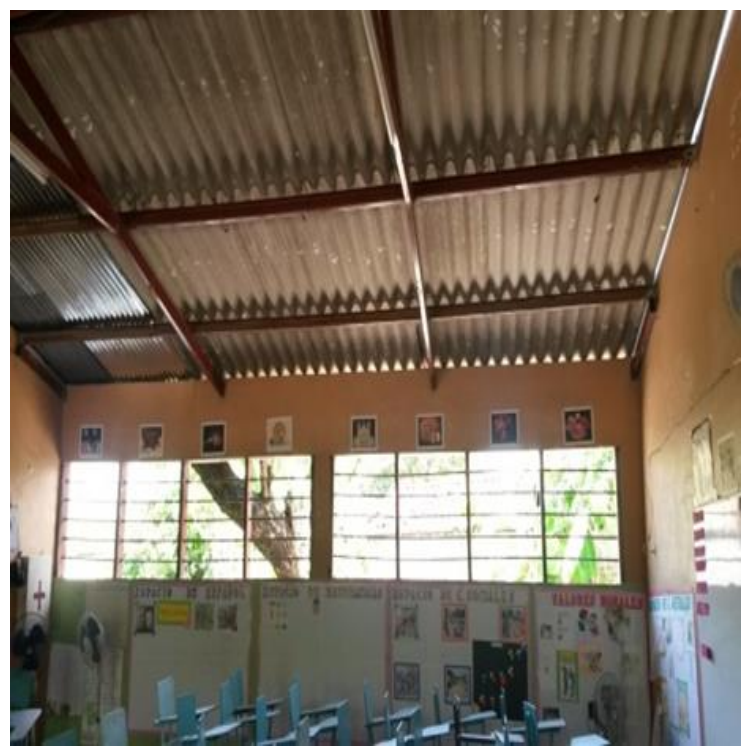

\section{Contrucción del Programa PROMINE}

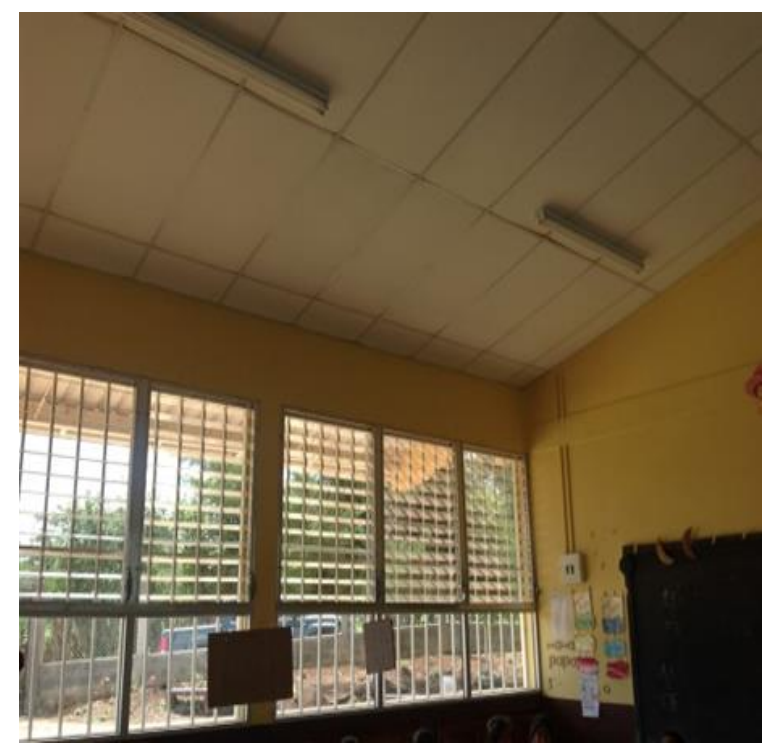

Figura 4. Diferencias entre aulas tradicionales y aulas con el sistema PEC-CPTEDBioclimático del Programa PROMINE/KfW

El Programa PROMINE/KfW, consideró mejorar el aula tradicional, implementando la metodología Bioclimática y CPTED para obtener un mejor ambiente dentro de las aulas de acuerdo a los climas de las zonas. La vigilancia natural desde el interior hacia el exterior se obtuvo a través de las actividades enunciadas en la Tabla 6.

Tabla 6. Tabla comparativa entre un proyecto tradicional y uno del Programa PROMINE-KfW

\begin{tabular}{|c|c|c|c|c|}
\hline & & Actividades & Tradicional & Por el Programa PROMINE \\
\hline \multirow{10}{*}{$\begin{array}{c}\text { Diferencias } \\
\text { entre las } \\
\text { Aulas }\end{array}$} & 1 & Ventanas & se usa balcón, & celosías y vidrio fijo \\
\hline & 2 & Balcón & tubo industrial & balcón de varilla \\
\hline & 3 & Cielo falso & no utiliza & $\begin{array}{l}\text { cielo falso horizontal o inclinado, depende del } \\
\text { clima }\end{array}$ \\
\hline & 4 & $\begin{array}{c}\text { moldura de cielo } \\
\text { falso }\end{array}$ & no utiliza & De madera \\
\hline & 5 & $\begin{array}{c}\text { Pintura de cielo } \\
\text { falso }\end{array}$ & no utiliza & Pintura acrílica \\
\hline & 6 & $\begin{array}{l}\text { Techo con aislante } \\
\text { térmico }\end{array}$ & $\begin{array}{l}\text { canaleta sin } \\
\text { aislante térmico }\end{array}$ & canaleta de 6" con aislante térmico \\
\hline & 7 & Pared de bloque & sisado sin repellar & repellada en el interior y exterior sisada \\
\hline & 8 & Pintura & $\begin{array}{l}\text { en elementos } \\
\text { tallados }\end{array}$ & $\begin{array}{l}\text { En pared repellada en el interior y exterior en } \\
\text { pared sisado y tallados. Pintura de aceite mate }\end{array}$ \\
\hline & 9 & Extractor Eólico & no utiliza & extractor eólico para clima caliente húmedo \\
\hline & 10 & Tronera & no utiliza & Incluir troneras en paredes en ambas culatas \\
\hline
\end{tabular}

\section{Conclusiones}

La inversión en infraestructura por parte del Programa PROMINE/KfW, está dando resultados satisfactorios que benefician simultáneamente a los centros escolares y a las comunidades, generándose conocimientos básicos para desarrollar y administrar proyectos de este tipo. De acuerdo a los resultados obtenidos de las encuestas realizadas, 
el mayor beneficiario de la inversión del programa es la comunidad, seguido por los alumnos. La capacitación orientada a las Sociedades de Padres de los Centros Educativos, producen un efecto positivo en la comunidad, motivando y acrecentando el interés en personas tanto jóvenes como mayores, para participar en el desarrollo del proyecto con la modalidad aprender haciendo, y volverlo así auto-sostenible.

Los montos de la inversión fueron definidos de acuerdo a la evaluación efectuada por la parte técnica del FHIS, conjuntamente con personal de la Secretaria de Educación. Para realizar las inversiones se consideraron las condiciones existentes en que se encontraban las instalaciones del centro y su red educativa, incluyendo los gastos operativos que inciden en el proyecto. Se consideraron ciertos factores relacionados con el predio y su entorno, el número de alumnos y maestros, aulas nuevas, reparaciones o ampliaciones que se ajusten a las normas de la Secretaria de Educación, etc. El monto de la inversión en los 25 centros seleccionados asciende a los 53.1 millones de Lempiras.

La infraestructura usando el sistema tradicional y la que utiliza metodologías PEC, Bioclimático y CPTED aplicadas por el programa PROMINE/KfW fueron comparadas. Las usadas por PROMINE/KfW presentan un mejor funcionamiento del centro y logran condiciones pedagógicas óptimas.

De los departamentos seleccionados por el Programa PROMINE/KfW, el departamento de La Paz es el que ha sido más favorecido, habiendo sido intervenidos hasta la fecha 9 Centros Educativos con 548 personas beneficiadas directamente y 178 empleos generados. El siguiente departamento más favorecido ha sido el de Intibucá con 466 personas beneficiadas y 120 empleos generados.

\section{Recomendaciones}

Elaborar un plan para el funcionamiento de relaciones entre los espacios arquitectónicos de un centro educativo ya sea existente, sujeto a remodelación, ampliación, como también de un nuevo proyecto, utilizando el Sistema Simplificado de Planeación Layout para lograr una distribución óptima de dichos espacios arquitectónicos.

Diseñar un Anteproyecto Arquitectónico de un Comedor-Cocina-Bodega con las áreas idóneas que cumpla con su función de servicio en donde se integre el Eco-fogón en el área destinada a la Cocina para la elaboración de la merienda escolar evitando la emanación de humo. Este proyecto puede ayudar al cuidado del medio ambiente, evitando el alto grado de consumo de madera y la tala de árboles.

La utilización de láminas traslúcidas para una mejor iluminación natural en el interior del Comedor y de la Cocina, incorporándose el Sistema Fotovoltaico de 130 watts para generar energía eléctrica para la noche. Dotar de un Sistema de captación de agua de lluvia en la cubierta del techo, que abastezca la demanda del Comedor-Cocina-Bodega durante el período escolar.

Es importante considerar aplicar la herramienta de Layout que se propone para el diseño de cualquier Centro Educativo, porque se obtendrán espacios arquitectónicos funcionales, y satisface las necesidades que el Ministerio de Educación desea. En relación al diseño arquitectónico del Comedor-Cocina-Bodega, beneficiará a una población estudiantil de cuarenta alumnos de Pre-básica y Básica. La utilización de la lámina traslúcida en las 
áreas de comedor y cocina mejorará la iluminación natural especialmente en las áreas de circulación, logrando aminorar el consumo de energía eléctrica en las instalaciones y así mismo se dará una reducción en el costo.

La recolección de agua de lluvia en los centros educativos vendrá a ser un modelo de conservación innovador en el país, mediante el cual se captará agua de lluvia para almacenarla y usarla en los períodos de escasez.

\section{Bibliografía}

Programa PROMINE/KFW, 2009. Acuerdo separado del contrato de Aporte Financiero y de Ejecución del Programa "Modernización de la Infraestructura Educativa y de su Gestión Local SE/FHIS VIII", de fecha 25 de junio del 2009, Aprobado por el KFW.

Convenio de Donación No 200866 178, suscrito entre el Banco Alemán de Desarrollo (KFW), Secretaria de Educación, y el Fondo Hondureño de Inversión Social (FHIS).

Crime Prevention through Environmental Design, (s. f.). Recuperado 29 de septiembre de 2013, a partir de http://www.crimewise.com/library/cpted.html

Gutierrez Vera, C., 2012. Guía para la aplicación bioclimática en escuelas rurales en Honduras.

KfW-FHIS, 2012. Manual de Proyectos Ejecutados por la Comunidad (PEC-FHIS) Programa Modernización de la Infraestructura Educativa y su Gestión local (PROMINE).

SEFIN, 2011. Informe de Cartera Alemania, Proyectos 31 Diciembre 2011. Recuperado de http://www.sefin.gob.hn/wp-content/uploads/2012/01/Informe-de-Cartera-KFWDic-2011.pdf

Modernización de la Infraestructura Educativa y de su Gestión Local SE/FHIS VIII, 2011. Elaborado por la Secretaria de información.

"LA REVISTA INNOVARE NO SE HACE RESPONSABLE EN NINGÚN CASO DE LOS CONTENIDOS, DATOS, CONCLUSIONES U OPINIONES VERTIDAS EN LOS ARTÍCULOS PUBLICADOS, SIENDO ESTA RESPOSABILIDAD EXCLUSIVA DEL (DE LOS) AUTOR (AUTORES)" 